\title{
An open-path, hand-held laser system for the detection of methane gas
}

\author{
Ben van Well†f, Stuart Murray§, Jane Hodgkinson\|, \\ Russ Pride\|, Rainer Strzoda $\uparrow$, Graham Gibson $\dagger$ \\ and Miles Padgett $\dagger$ \\ $\dagger$ Department of Physics and Astronomy, University of Glasgow, Glasgow, G12 8QQ \\ $\S$ AOS Technology Ltd, Melton Mowbray, Leicestershire, LE13 0RG \\ || Advantica Technologies Ltd, Loughborough, LE11 3GR \\ ฯ Siemens AG, Otto Hahn-Ring 6, ZT KM2, D-81730, Munich
}

\begin{abstract}
We have developed an open-path hand-held gas detector incorporating a distributed feedback InGaAs laser diode at $1.65 \mu \mathrm{m}$. Incorporated into a handheld transceiver unit, the emitted laser beam is backscattered from nearby surfaces, collected and focussed onto an amplified InGaAs detector using a $150 \mathrm{~mm}$ diameter plastic Fresnel lens. At ranges of 4-5metres, a typical backscattered signal is 10's of nanowatts of laser light. Applying 2nd derivative wavelength modulation spectroscopy gives a sensitivity to methane of better than $10 \mathrm{ppm}$ over a one metre path length. A number of demonstration units have been fabricated and successfully evaluated by end users.
\end{abstract}

E-mail: b.vanwell@physics.gla.ac.uk 


\section{Background}

The low-cost, sensitive detection of methane gas has wide use amongst the gas utility companies for both routine pipeline inspection and leak-report response applications [1]. The conventional approach to leak detection is based upon flame ionisation detectors [2] (FID) but such technology measures concentration at only a single point. Using a point sensor is problematic in that the leak may be overlooked, the user may have to enter potentially explosive situations and that above-ground pipe-work could be difficult to access. This paper details the design and operational performance of an optical instrument capable of measuring methane at a range of several metres with a sensitivity close to that required for detecting the atmospheric background of $1.6 \mathrm{ppm}$.

A variety of instruments based on optical absorption of light can be configured to detect gas over the line of sight of a light beam. Sophisticated LIDAR instruments [3] have been reported using pulsed-lasers, tuned to the resonant frequency of the target gas, where backscattered light from the gas in the atmosphere gives both gas concentration (from the size of the signal) and range (from the delay time). Such instruments are usually vehicle or aircraft based, are rarely eye-safe and have a cost/complexity that limits their wide-spread use. Our approach has been to dispense with the need for range information and rely on the deliberate backscattered signal obtained from the ground or building over a range of only several metres, thereby giving a larger continuous signal, detectable with a lower cost/complexity instrument.

\section{Operating principles of the optical gas detector}

Methane gas has its strongest absorption in the 3 micron spectral region, but corresponding laser sources, including cryogenically cooled laser diodes and optical parametric oscillators, exceed the cost budget for a hand-held system. Methane has an additional absorption $\nu_{3}$ band at 1.6 microns which, although over one order of magnitude weaker, does coincide with the single-mode, single-frequency emission wavelength of InGaAs distributed feedback laser diodes [4]. Furthermore, diode lasers are readily temperature-tuned to match the absorption wavelength, and subsequent control of their drive current gives fine scale tuning and the option of wavelength modulation. InGaAs diode lasers can be readily tuned over a few percent of their operating wavelength by changing their temperature and over several $\mathrm{GHz}$ by changing the drive current. However, day-to-day variation in their tuning parameters means that absolute wavelength stability is problematic - and a wide wavelength scan of a gas filled reference cell is required. Remote hand-held gas detection instruments have been reported previously [5], but our incorporation of a dedicated laser module with internal reference cells means that we are assured that the laser is operating at the correct wavelength and that the integrity of the zero gas signal is assured - a key requirement in all safety critical environments. 
Given a tuneable laser source, a number of spectroscopic techniques can be applied to the detection of gas, including, photo-acoustics [6], cavity ring-down [7], amplitude, wavelength or frequency modulation [8]. Although highly sensitive to small absorptions, photo-acoustic and cavity ring-down techniques require dedicated sample cells and are therefore not suited to open-path applications. Using a laser tuned exactly to the absorption wavelength of the gas could be used to monitor the absorption and hence gas concentration but in our configuration a change in detected power could also arise from a change in range or backscattering surface, rather than gas concentration. It is common practice in such situations to rely on a rapid modulation of the laser frequency/wavelength in the neighbourhood of the gas absorption giving a corresponding modulation in the detected power, the magnitude of which is related to the gas concentration. When the modulation frequency is comparable to the modulation depth and large compared to the width of the gas absorption, the technique is called frequency modulation spectroscopy. When the modulation frequency is small compared to the modulation depth and width of gas absorption, the technique is called wavelength modulation spectroscopy [9]. Both techniques have been widely implemented [10] and are capable of measuring small absorptions with a sensitivity approaching that limited by the shot noise fluctuations in the power of the laser itself. For the spectroscopy of gases at atmospheric pressure, the absorption width typically approaches $1 \mathrm{GHz}$, meaning that the modulation and demodulation required for frequency modulation spectroscopy is technologically challenging. Consequently, wavelength modulation is the natural choice as a spectroscopic technique for detection of gases at atmospheric pressure.

Current modulation of the laser wavelength over the absorption line of the gas results in a modulation of the detected laser power, the phase and amplitude of which is readily measured using a lock-in-amplifier. The amplitude of the $n^{\text {th }}$ harmonic of the modulated power is proportional to the $n^{\text {th }}$ derivative of the detected power. Within our instrument we use first, second and third harmonic demodulation to give respectively, the first, second and third derivatives of the absorption as a function of wavelength. Note that, of these harmonics it is the second derivative that has a maximum value at the wavelength corresponding to the centre of the absorption feature.

\section{Our instrument design}

Our instrument is configured as an optical head containing laser diode module, temperature control electronics, collection lens and photodiode. This is coupled to a separate control box containing the microprocessor control, analogue to digital interface, laser diode driver and multiple lock-in-amplifiers/phase shifters. A photo of the complete instrument is shown in figure 1 .

The laser diode module is mounted in a standard 14 pin butterfly package incorpo- 

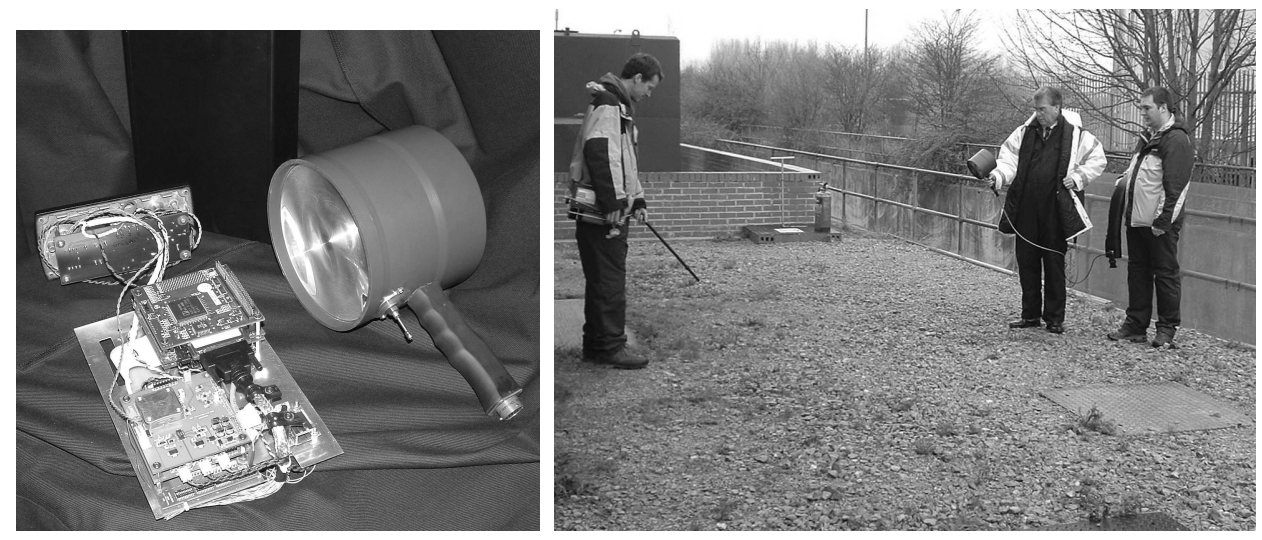

Figure 1. Left: Complete instrument package, Right: Field testing of prototype instrument

\section{Top View}

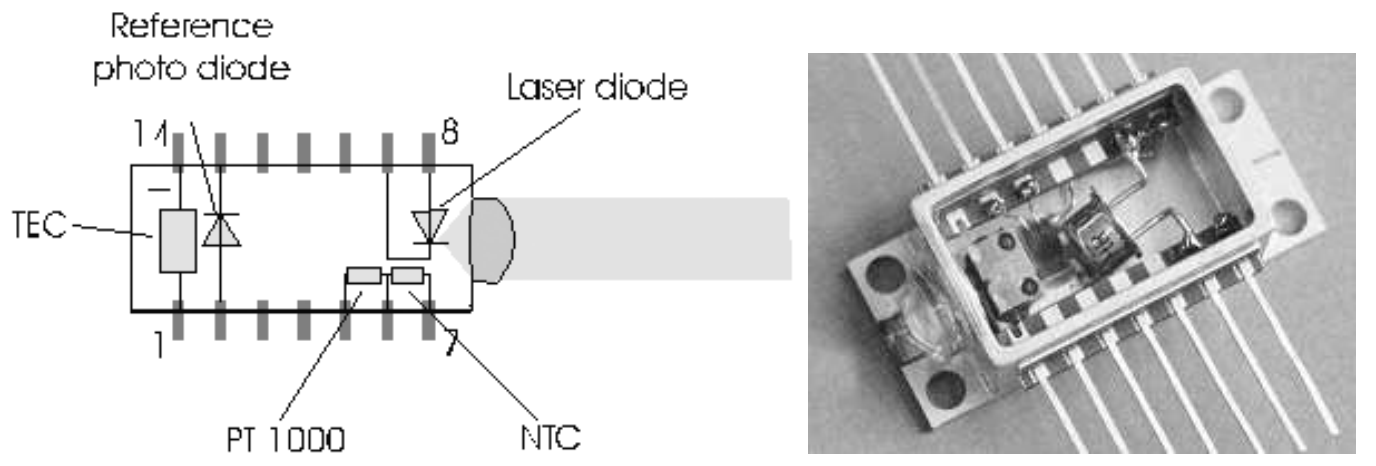

Figure 2. Completed laser diode module package

rating the $1651 \mathrm{~nm}$ laser diode mounted on a thermoelectric cooler, a $4.5 \mathrm{~mm}$ focal length aspheric lens to collimate the emission from the front facet, and a separate photodiode monitoring the rear facet emission. The commercially available monitor photodiode package (TO) is filled with methane gas, forming a reference cell from which the operating wavelength of the laser can be controlled. The $1 \mathrm{~mm}$ thickness of gas between the diode and package window gives an absorption of approximately $4 \%$ at $1651 \mathrm{~nm}$. The optical layout and photo of complete laser module is shown in figure 2.

The near-collimated laser beam emerges from the optical head and is backscattered from any object in the beam. The backscattered light is collected using a $150 \mathrm{~mm}$ diameter, $80 \mathrm{~mm}$ focal length, plastic Fresnel lens that focuses the light onto a $1 \mathrm{~mm}$ diameter InGaAs signal photodiode with integral preamplifier. This detector is shielded from excess sunlight, which would otherwise saturate the amplifiers, by a 100nm bandwidth filter centred at $1680 \mathrm{~nm}$. Typically when pointed at a wall or ground at a distance of 3-5metres the backscattered signal is of the order of 10-100 $\mathrm{nW}$ compared to the noise 


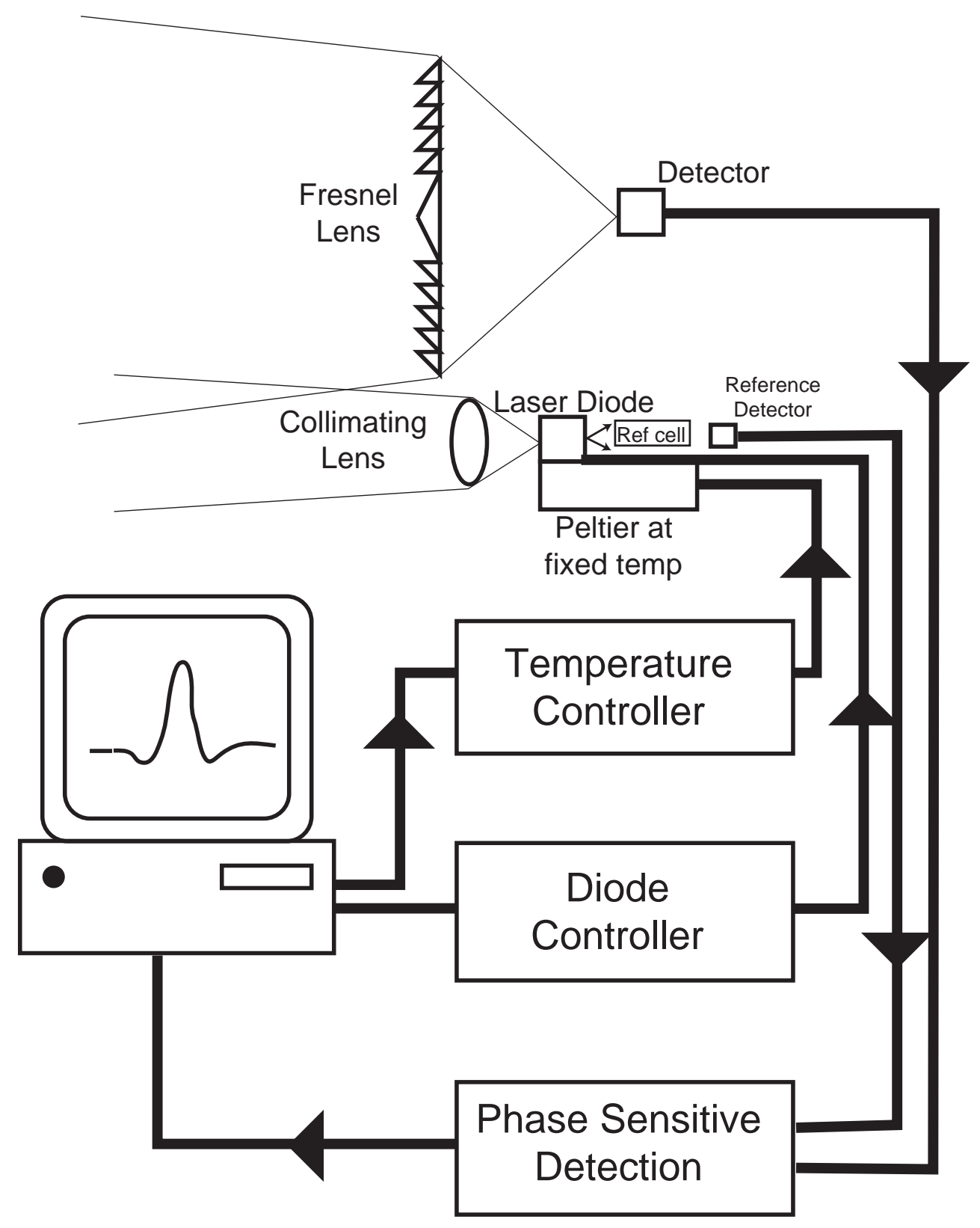

Figure 3. Schematic diagram showing signal path through the system

equivalent power of the detector of $2.9 \mathrm{pW} / \sqrt{\mathrm{Hz}}\left(1.5 \times 10^{6} \mathrm{~V} / \mathrm{A}\right.$ gain, $3 \mathrm{~Hz}$ bandwidth $)$.

The laser diode driver (Wavelength Electronics) is housed within the shoulderstrapped control box and the laser current drive is fed to the optical head via a shielded multi-core cable, which also feeds the amplified signals from both monitor and signal photodiode back to the control box. The laser diode driver supplies a DC current of approximately $70 \mathrm{~mA}$ giving a laser output power of around $8 \mathrm{~mW}$. Superimposed upon this DC level is a $6 \mathrm{kHz}$ modulation with an amplitude of $6 \mathrm{~mA}$, sufficient to modulate the laser wavelength over the width of the methane absorption. Within the control box, 
the $\mathrm{DC}$ voltage from the monitor photodiode is read directly by the microprocessor to confirm laser operation.

The photodiode signals are demodulated at various harmonic frequencies using Analogue Devices AD630 integrated circuits. The monitor photodiode signal is demodulated at both 12 and $18 \mathrm{kHz}$. The $12 \mathrm{kHz}$ demodulated signal corresponds to the second-derivative of the absorption, a signal which is a maximum and symmetric about the centre of the absorption feature, and is used to confirm the operating wavelength of the laser. The $18 \mathrm{kHz}$ demodulated signal corresponds to the third derivative, a signal which is zero and anti symmetric about the centre of the absorption feature, providing an error voltage enabling the software to make fine adjustments to the laser wavelength, maintaining it exactly at the absorption feature.

The signal photodiode is demodulated at 6 and $12 \mathrm{kHz}$. The $6 \mathrm{kHz}$ demodulated signal is dominated by the offset corresponding to the linear dependence of laser power on drive current and acts as an accurate measure of the received laser power independent of any residual sensitivity to sunlight or other thermal sources. A lower limit is set upon this detected laser power such that below a certain threshold the software gives a "low-light" warning. The $12 \mathrm{kHz}$ demodulated signal gives a measure of the methane gas within the line of sight of the instrument. The normalised gas concentration reading is obtained from the ratio of the $12 \mathrm{kHz}$ to the $6 \mathrm{kHz}$ signal.

The whole instrument is under micro-processor control in the form of a board level personal computer (NS Geode $300 \mathrm{MHz}$ ), operating under Windows XP Professional and running control software written within the LabVIEW programming environment. Upon power-up, the computer auto boots and launches the control software. The control software maintains all aspects of instrument operation, including start-up sequence, locking the laser wavelength to the absorption feature, and calculating a gas concentration signal which is displayed on a digital readout on the back surface of the optical head. The output stage associated with all these demodulation circuits is set to a time constant of $10 \mathrm{msec}$, although additional filtering with the control software typical increases this to $300 \mathrm{msec}$ - an ideal response time for user interaction. During operation, various signal levels are monitored with respect to confirming laser operation, wavelength lock and that there is sufficient backscattered light to make an accurate reading. Failure in any of these aspects results in a specific error-code on the gas reading display. Figure 4 shows a flow diagram of the software control program.

\section{Laboratory characterisation of the instrument performance}

When operating within the laboratory, the control software is configured to scan the laser wavelength over the absorption feature and record and display the various spec- 


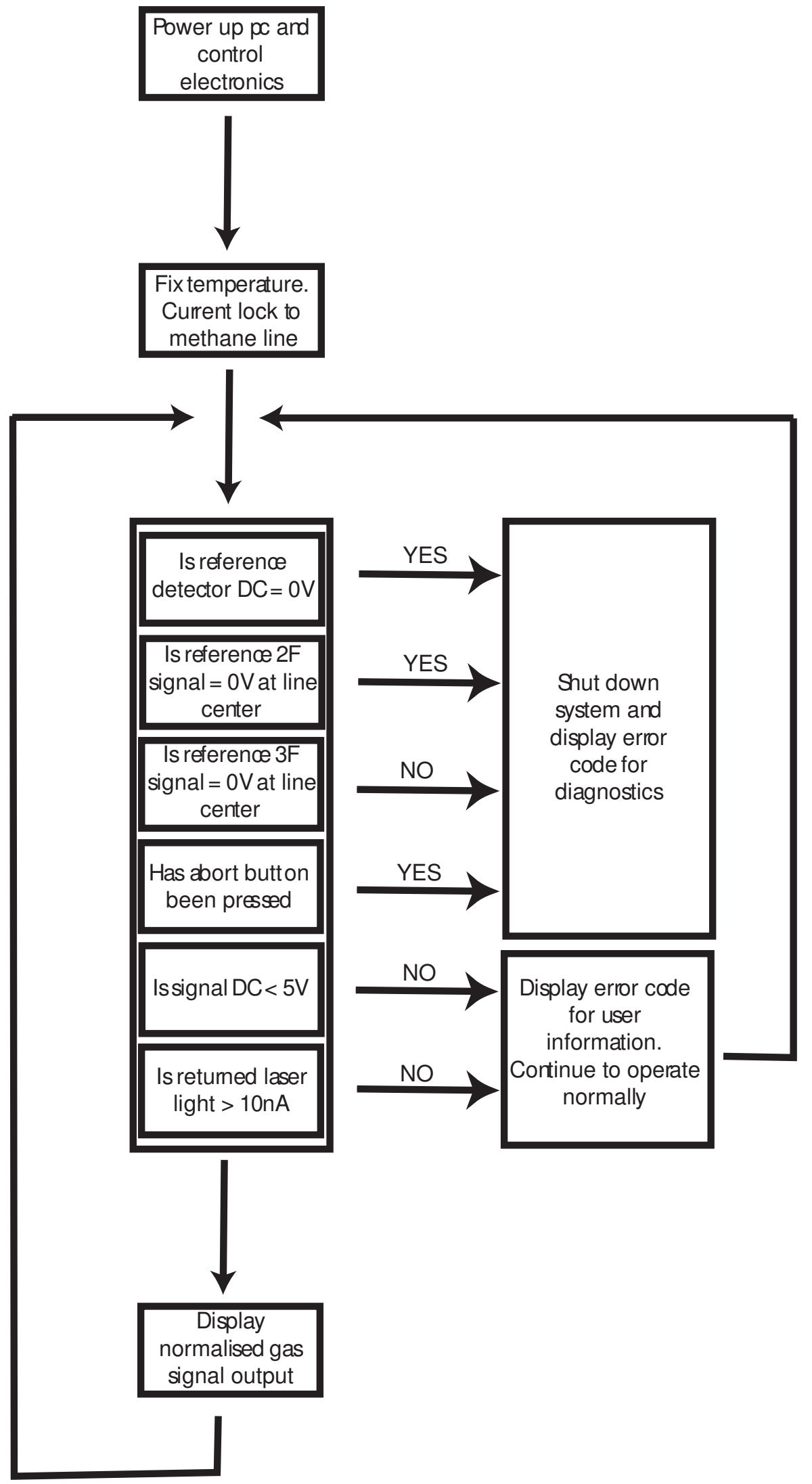

Figure 4. Flow diagram of software control 

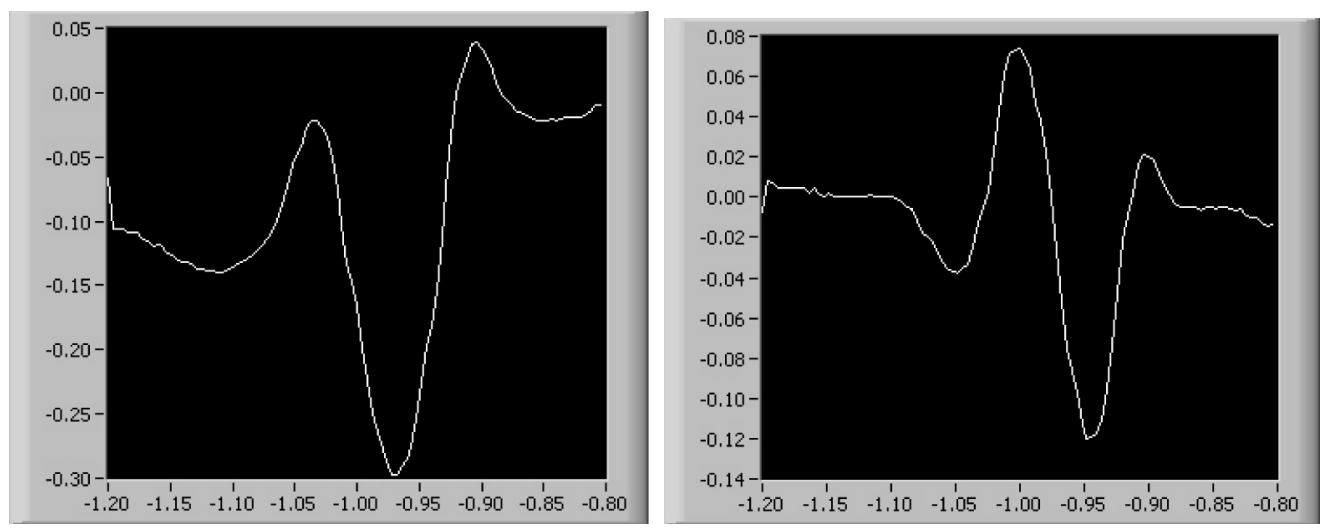

Figure 5. Second (left) and third (right) harmonic demodulated spectra recorded from the reference detector
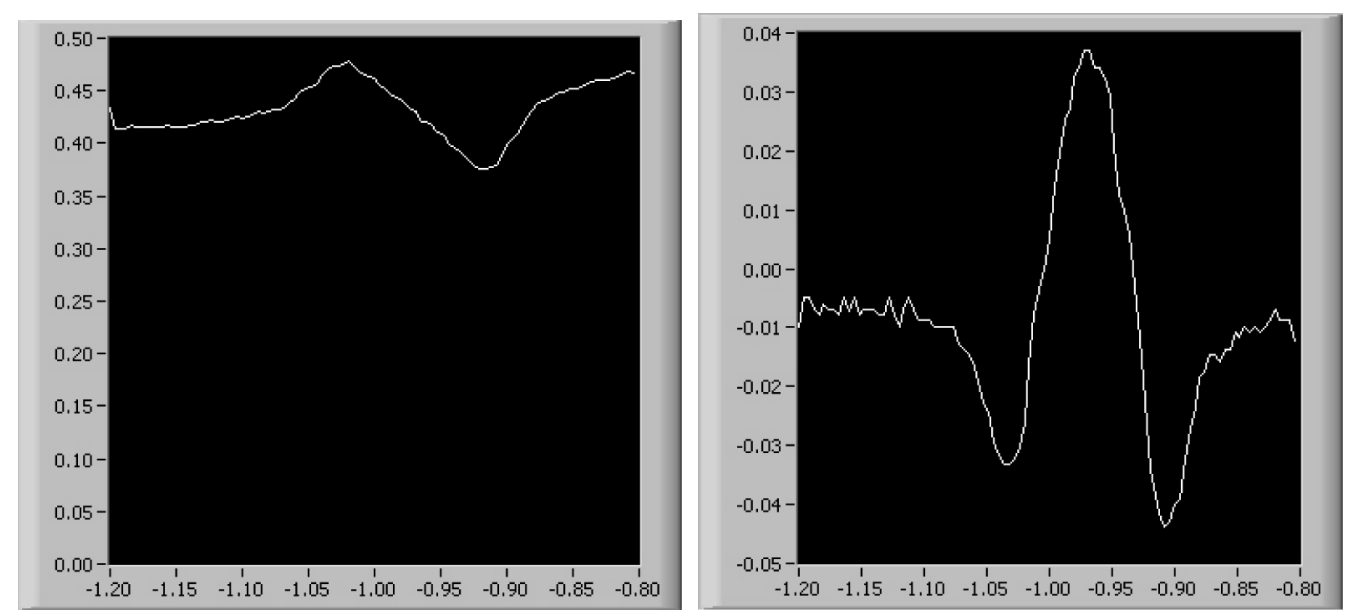

Figure 6. First (left) and second (right) harmonic demodulated spectra recorded from the signal detector

tral signals on an external monitor. Figure 5 shows typical traces obtained for the demodulated signals on the reference channel. Figure 6 shows typical traces from the demodulated waveforms from the signal photodiode.

Those signals obtained from the reference photodiode are independent of gas in the atmosphere and show clearly the characteristic second and third derivative spectra. The signals from the signal photodiode correspond to a received power of $30 \mathrm{nW}$ and a gas concentration of $1 \%$, confined to a $100 \mathrm{~mm}$ length of the beam, i.e. 1000ppm.metre. The key parameter in determining the smallest amount of gas that can be measured is the amount of backscattered light reaching the detector. Figure 7 shows a graph of the minimum detectable gas concentration ( $\mathrm{S}: \mathrm{N}$ 1:1) as a function of received power. The detection limit scales inversely with the returned light power, indicating that the system has a constant noise floor. This is approximately 2-3 times greater than that predicted by the NEP of the detector. 


\section{Sensitivity vs. Photocurrent}

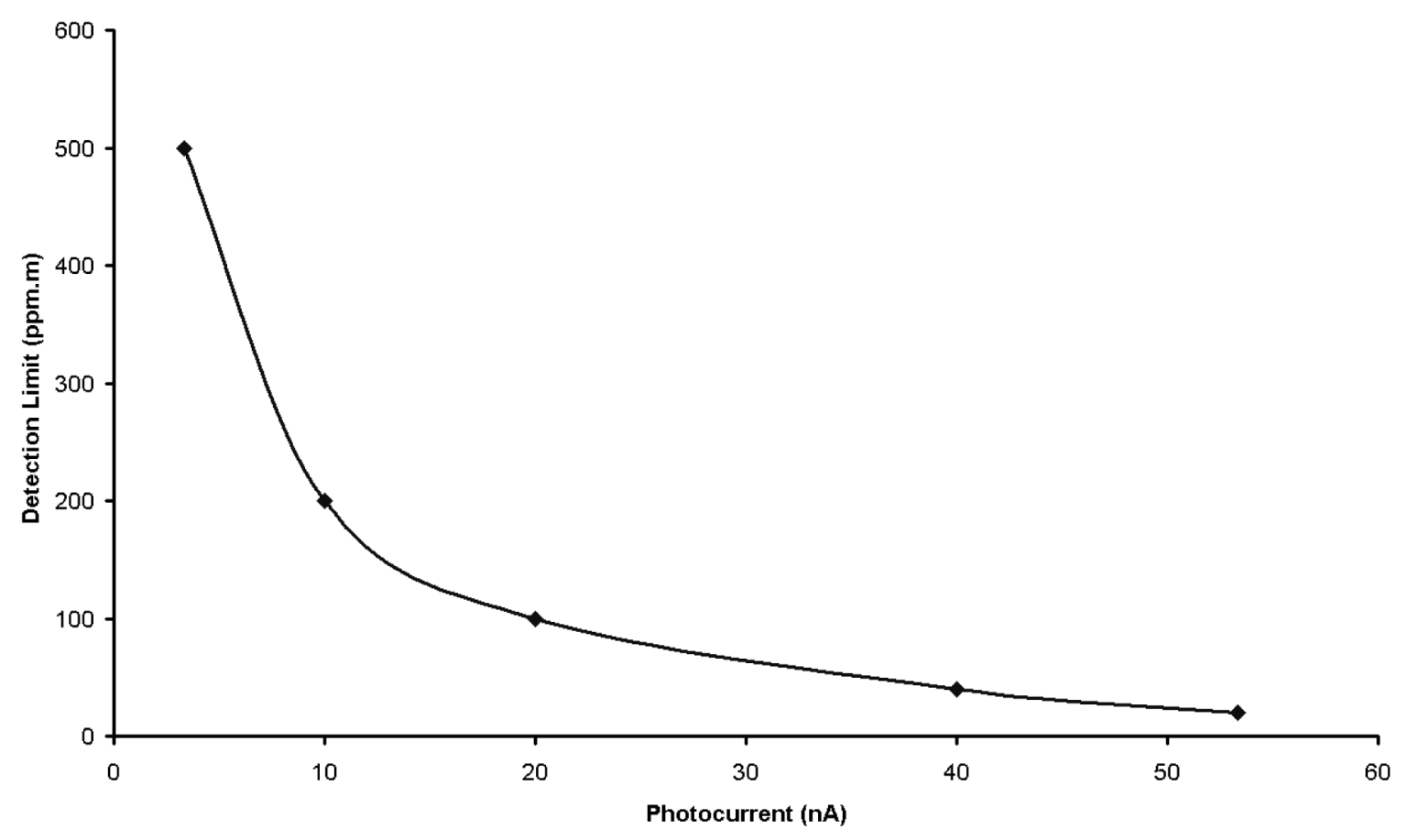

Figure 7. Minimum detectable gas concentration for a given light level

\section{Field-testing and evaluation of the instrument}

For outdoor use, the control software is configured to maintain the laser wavelength at exactly the absorption feature. The time constant of the demodulation is set to $300 \mathrm{~ms}$, updating the gas concentration output about three times per second. A low $(<10 n W)$ received light level triggers a negative concentration display of -9000 ppm.metre and a sunlight saturation of the detector as a negative display of $-8000 \mathrm{ppm}$.metre. Most of the instrument characterisation was carried out at an open air test site licensed to undertake such work.

The key comparison is to demonstrate that the optical instrument can give similar reading to a FID instrument whilst given the additional advantage of remote sensing. Using a manufactured leak buried at a realistic depth of $80 \mathrm{~cm}$ beneath the ground we invited a FID user to "patrol" a defined area, looking for signs of a gas signal. At all times, standing at a distance of 4 metres, we directed the beam of the laser light from the optical gas detector at the FID inlet. Figure 8 shows the readings from the two types of instrument plotted as a function of time. Although not meaningful to compare the point concentration measured by the FID with the integrated over path concentration measured by the optical instrument directly, we see that the general form of the two signal is extremely similar. A series of field testing by end users have reported similar 
FID Instrument

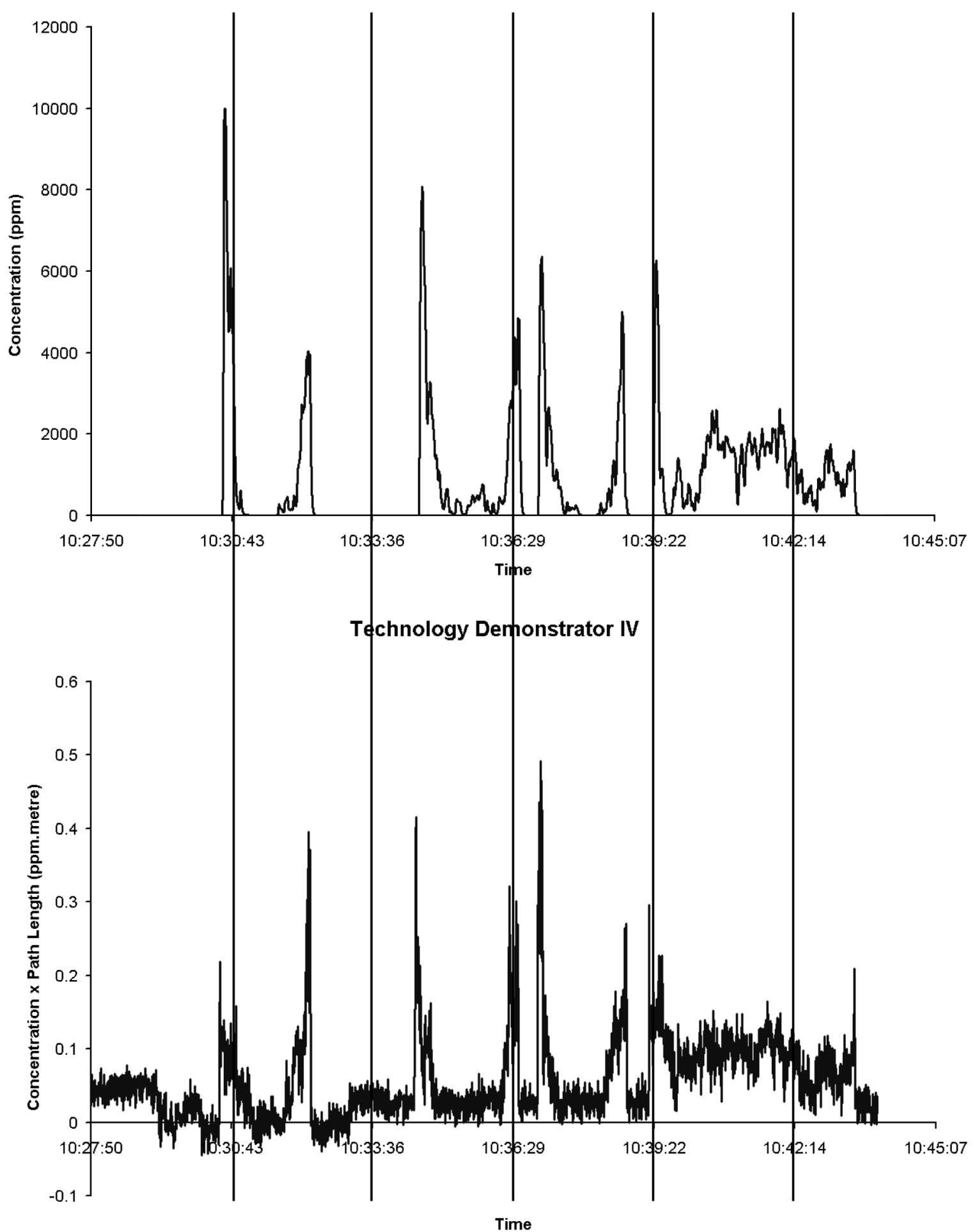

Figure 8. Comparison between a FID and the remote gas detection system comparisons. 


\section{Discussion}

The close equivalence of the reading from the FID and optical instrument suggest that the optical instrument could be used in the field as a direct replacement of the FID approach. Furthermore the remote sensing capability of the optical instrument opens new modes of use such that the operator can inspect a location that is difficult to access, whether on grounds of geometry or safety.

\section{References}

[1] HSE Books, A Guide To Gas Safety (Management) Regulations, 1996

[2] Simpson, 1981, Direct quantitative-analysis using flame ionization detection - the construction and performance of the fidoh detector, J. Chromatogr. Sci. 19 275-282

[3] Walmsley, 1998, The accuracy and sensitivity of infrared differential absorption lidar measurements of hydrocarbon emissions from process units, Pure and Applied Optics 7 907-925

[4] Temkin, 1990, InGaAs/InP distributed feedback quantum well laser, Applied Physics Letters $\mathbf{5 7}$ $1295-1297$

[5] Iseki, 2000, A portable remote methane sensor using a tunable diode laser, Meas. Sci. Technol. 11 594-602

[6] Zelinger, 2000, Quantitative analysis of trace mixtures of toluene and xylenes by CO2 laser photoacoustic spectrometry, Analytica Chemica Acta 422 179-185

[7] Paldus, 1998, Cavity-locked ring-down spectroscopy, Journal of Applied Optics 83 3991-3997

[8] Schilt, 2003, Wavelength modulation spectroscopy: combined frequency and intensity laser modulation, Applied Optics 42 6728-6738

[9] J. M. Supplee, E. A. Whittaker and W. Lenth, 1994, Theoretical description of frequency modulation and wavelength modulation spectroscopy, Applied Optics 33 6294-6302

[10] Bomse, 1992, Frequency modulation and wavelength modulation spectroscopies: comparison of experimental methods using a lead-salt diode laser, Applied Optics 31 718-731 